\title{
PHOTONIC RESERVOIR COMPUTING USING DELAY DYNAMICAL SYSTEMS
}

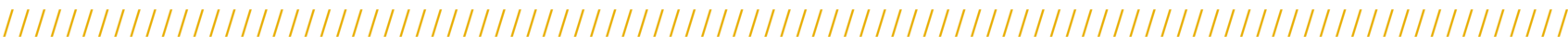

\section{Piotr ANTONIK ${ }^{1}$, Serge MASSAR ${ }^{2, *}$, Guy VAN DER SANDE ${ }^{3}$}

${ }^{1}$ LMOPS EA 4423 Laboratory \& Chair in Photonics, Centrale-Supélec \& Université de Lorraine, 2 rue Edouard Belin, Metz, France

${ }^{2}$ Laboratoire d'Information Quantique, CP224, Université libre de Bruxelles, Av. F. D. Roosevelt 50, Bruxelles, Belgium

${ }^{3}$ Applied Physics Research Group, Vrije Universiteit Brussel, Pleinlaan 2, Brussels, Belgium

*smassar@ulb.ac.be

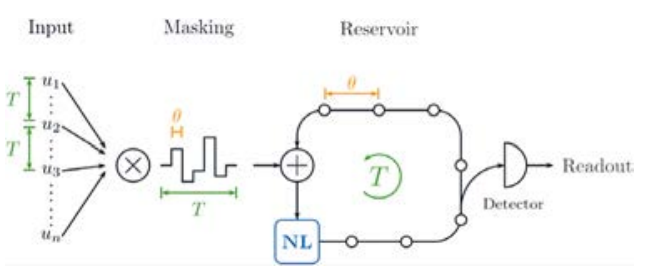

The recent progress in artificial intelligence has spurred renewed interest in hardware implementations of neural networks. Reservoir computing is a powerful, highly versatile machine learning algorithm well suited for experimental implementations. The simplest highperformance architecture is based on delay dynamical systems. We illustrate its power through a series of photonic examples, including the first all optical reservoir computer and reservoir computers based on lasers with delayed feedback. We also show how reservoirs can be used to emulate dynamical systems. We discuss the perspectives of photonic reservoir computing.

https://doi.org/10.1051/photon/202010445

\section{NEURAL NETWORKS}

The next decades will see dramatic evolutions in information processing technology. The exponential improvements of computers known as Moore's law is expected to end. Therefore, in order for performance to continue to improve, one will have to devise computing systems based on entirely new architectures and/or components. One of the most promising approaches is to take inspiration from the biological brain. The brain is able to do remarkable computations, whose underlying algorithm we do not understand, with very low footprint and energy consumption. The brain's architecture is completely different from the von Neumann architecture used in digital computers. It is based on a very large number $\left(10^{10}\right)$ of slow (ms time scale) neurons, massively connected together, working in parallel. Thus, an alternative exists.
During the last decades artificial intelligence has made dramatic progress. It enables computers to equal or surpass humans at tasks such as image recognition or game playing. These algorithms are most often based on artificial neural network (i.e. brain inspired). They can be viewed as a group of interconnected nonlinear nodes (neurons) on which input signals are applied.

Because such artificial neural networks are fundamentally analog systems, one expects that significant savings in energy consumption and footprint could be achieved by implementing them in analog, rather than digital, hardware. This old dream is attracting renewed interest. Photonics is a promising area in which to realize such analog neural networks. Indeed, photonics allows one to implement high-performance systems and can in principle allow for much higher speed of operation than electronics, with a high degree of multiplexing. Photonic information processing systems would find a natural area of application in telecommunications. It is still too early to know if such promises will be realised. In the following, we sketch some recent work on photonic

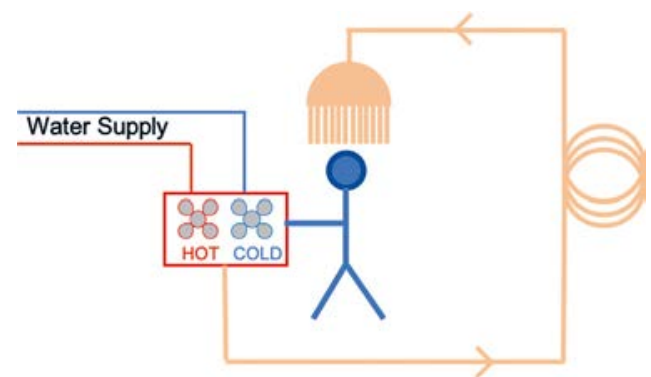

Figure 1.

Schematic of a delay dynamical system. The nonlinear node is the sensory and motor system of the person under the shower. The delay is induced by the pipes between the faucets and the shower. 
neural networks based on the reservoir computing paradigm.

\section{RESERVOIR COMPUTING}

In general, it is very difficult to optimize all the links (inputs to neurons, interconnections between neurons, neurons to output) in a neural network. Indeed, the most general neural networks are recurrent networks, i.e. networks that have feedback loops. But training recurrent networks is hard, because, as one changes the interconnection weights of a recurrent network, one may suddenly enter new dynamical regimes. The system may start oscillating or become chaotic. Such changes completely modify the behaviour of the network and severely impair its information processing capability. Training mechanisms cannot cope with such brutal changes. Therefore, most neural networks use simpler architectures. For instance the deep learning neural networks behind the recent dramatic progress in artificial intelligence are based on feed forward networks without any recurrences.

Reservoir computing [1] uses recurrent networks, but in a way that circumvents the above problems. In this approach, the interconnections between neurons are fixed and chosen randomly. This network of connected neurons, called the Reservoir, is driven by a time-dependent signal. The strength of the connection between neurons is adjusted by a global scaling to a regime where the network will respond deterministically, but in a nonlinear way, to the input signal. The only part of the reservoir computer that is trained is the output layer, i.e. the connection between the reservoir and the output neurons. This training is easy because there is no feedback between the output and the reservoir. For instance, one can drive the reservoir with a speech signal, and train an output neuron to have a high level (corresponding to 1 ) if a certain word is pronounced, and to have a low level (corresponding to 0 ) otherwise. This approach is not as powerful as the deep networks mentioned above and is no longer the state of the art in machine learning. But it does have very good performance on tasks such as speech recognition, time series prediction, etc.

Reservoir computing is a flexible approach. The fact that the networks can be chosen randomly means that there is no need to use biologically realistic neurons. Most high dimensional dynamical systems should work, provided the strength of the nonlinearity can be tuned to bring the system slightly below the threshold for spontaneous oscillations or chaos. This flexibility makes reservoir computing particularly well suited for experimental study.

\section{DELAY DYNAMICAL SYSTEMS}

Delay dynamical systems can be found in biology, chemistry, physics, engineering. A simple example is the regulation of the temperature in a shower, see Fig. 1. Imagine you are taking a shower, and the water is too cold. You turn the hot water faucet. There is a delay between your action on the faucet, due to the length of pipe between the faucet and the shower, and the change in temperature of the water. If you have turned the faucet too far, the water may become burning hot after that delay. So you switch off the hot water faucet. Now, it is freezing cold. If you continue, you may oscillate indefinitely between a too hot and a too cold shower, without ever reaching the right temperature.

This is a typical example of a delay dynamical system. Your action on the system at time $t$ depends on its state at an earlier time $\mathrm{t}-\mathrm{T}$ (here $\mathrm{T}$ is the delay induced by the pipes). As the example shows, such systems can have highly complex dynamics. They can exhibit oscillations and even chaos. Furthermore, they are very high dimensional systems, since the state of the system at time $t$ depends on all the past history between $t$ and $t-T$.

\section{Figure 2 .}

All-optical reservoir computers based on delay dynamical systems. Left, schematic of the first all-optical reservoir computer [3] operating in incoherent light. Right, schematic of a reservoir computer based on a semiconductor laser with delayed feedback [4]. In both experiments the masked input is encoded using a Lithium Niobate Mach-Zehnder intensity modulator (MZ). Fiber optics components are in orange. The nonlinear node which is essential for in reservoir computers is represented in blue. Super-luminescent Light Emitting Diode (SLD), Attenuator (Att), Semiconductor Optical Amplifier (SOA), Laser Diode (LD).
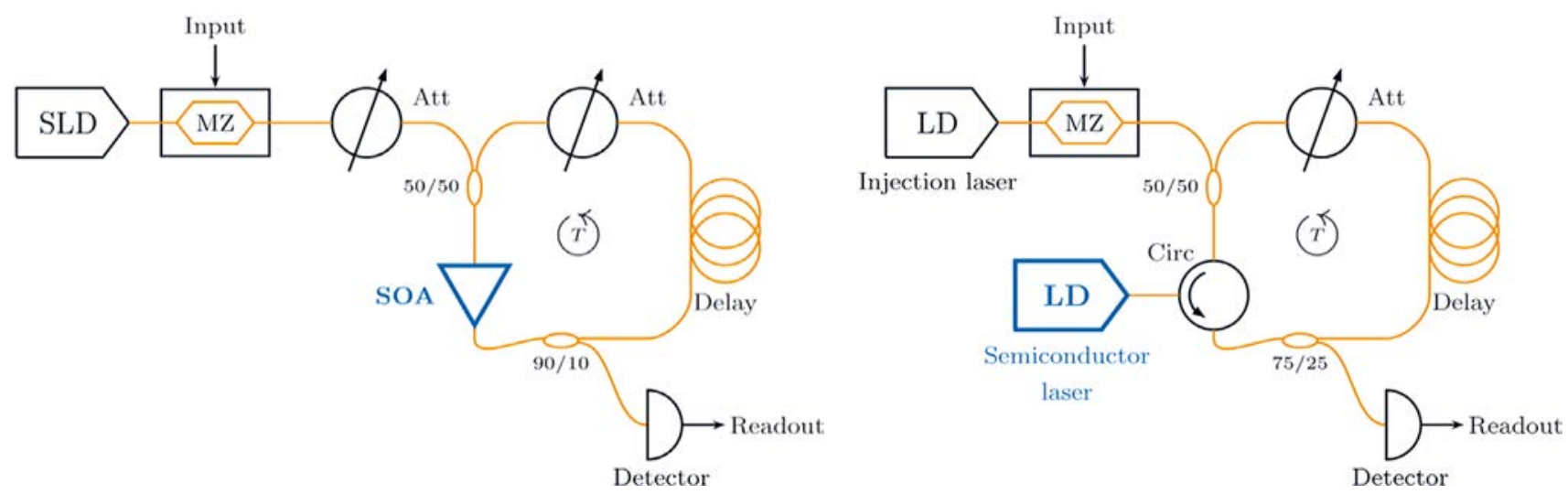
Delay dynamical systems are rather easy to implement and study experimentally, because they require only a delay loop and a nonlinear node through which the feedback acts. In the example, the delay is the pipe, and the nonlinear node is the combination of your sensory and motor system.

\section{DELAY SYSTEMS AS RESERVOIRS}

The rich dynamics, high dimensionality, and ease of experimental implementation make delay dynamical systems good candidates for reservoir computers. How does one transform a delay dynamical system into a reservoir? The answer is to divide the delay loop of length $\mathrm{L}=\mathrm{vT}$ (with $\mathrm{v}$ the speed of propagation around the loop) into $\mathrm{N}$ intervals of length $\mathrm{L} / \mathrm{N}$ and duration $\theta=\mathrm{T} / \mathrm{N}$, and consider each interval as a "neuron" [2]. In this way, we create a large number $\mathrm{N}$ of neurons (typically $\mathrm{N}$ ranges between a dozen to several hundreds). We then drive the delay system with the input signal $u$ to which we apply a sample and hold procedure so that the input is held constant during a delay $\mathrm{T}$.

However, this procedure is not sufficient, because all the $\mathrm{N}$ neurons would be identical, all having the same behaviour. To solve this issue, a masking procedure is applied: the input $\mathrm{u}$ is multiplied by a mask $\mathrm{m}(\mathrm{t})$ which is periodic of period $\mathrm{T}$. In this way, each neuron is multiplied by a different value of the mask, and therefore exhibits different dynamics. The Table of Content Graphics represents schematically the masking procedure and the neurons distributed along the delay loop.

In order to further improve performance it is necessary to couple the neurons together. Two solutions have been proposed. The first is to introduce a low pass filter in the delay loop with time constant comprised between $\mathrm{T}$ and $\theta$. The second is to slightly desynchronize the time during which the input is held constant and the round-trip time T.

The output of the reservoir is obtained by measuring the system during a time $\mathrm{T}$, thereby collecting the states of the $\mathrm{N}$ neurons. The output is a linear combination of these $\mathrm{N}$ states, with the weights of the linear combination adjusted through a training procedure.

\section{ALL-OPTICAL RESERVOIR COMPUTER}

These principles were used to build the first all optical reservoir computer [3]. It consists of a fibre optics delay dynamical system operating in the telecommunication C-band, see Fig. 2. This first system operated using incoherent light. An electronic signal corresponding to the time dependent input multiplied by the input mask is generated electronically and drives an intensity modulator thereby producing a time dependent input optical signal whose intensity is adjusted with a variable attenuator and which is then is injected into the fiber loop. The delay dynamical system consists of a Semiconductor Optical Amplifier (SOA), a variable optical attenuator, and a fiber spool that acts as delay line. The SOA operating near saturation acts as nonlinear node. The cavity operates below the lasing threshold. A tap coupler followed by a readout photodiode is used to measure the neurons. The round trip time of the delay system was $\mathrm{T}=7.9 \mu \mathrm{s}$, corresponding to the time needed to process one input. The number of internal nodes, i.e. neurons, was varied between $\mathrm{N}=50$ to 200 . The output of the reservoir was computed off-line on a digital computer. The system was used for a telecommunication inspired task, the equalization of a nonlinear communication channel, and for a simple speech recognition task, the recognition of isolated spoken digits.

\section{RESERVOIR COMPUTER BASED ON SEMICONDUCTOR LASER WITH DELAYED FEEDBACK}

Semiconductor lasers are the most common type of lasers, with a wide range of applications such as telecommunication, material processing, etc. Semiconductor lasers submitted to optical feedback have a rich dynamic which has been intensively studied. In reference [4] the nonlinear response of a semiconductor laser subject to feedback was used as reservoir, see Fig. 2. The external input was injected as a modulated optical field and the output layer was implemented off-line after detection. The optical feedback loop had a delay of $\mathrm{T}=77.6$ ns. It was used to classify spoken
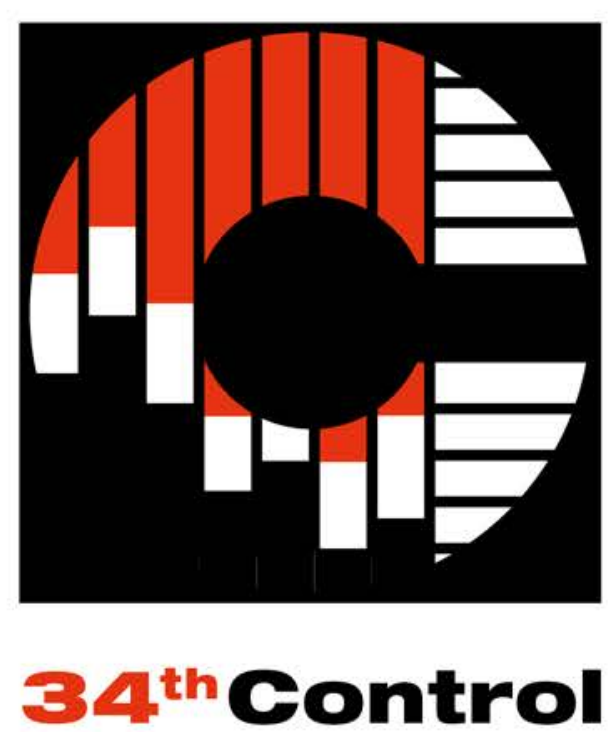

\section{International trade fair for quality assurance}

\section{曲}

P Stuttgart

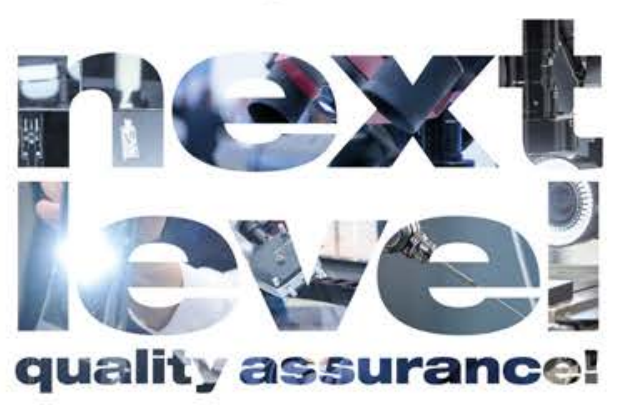

industrial image processing multi sensor = vision systems embedded vision = QA software - artificial intelligence - machine learning - 3d-metrology - additive manufacturing - hyperspectral imaging - microscopy - endoscopy - heat flow thermography - ultrasound - magnetic resonance - X-ray CT = OCT - ellipsometry - polarization - associated components - precision measurement - real-time data quality networking = QA systems

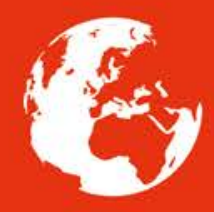

The world's leading trade fair

for quality assurance

4) www.control-messe.com

L. \#controle021 in If 0 . 

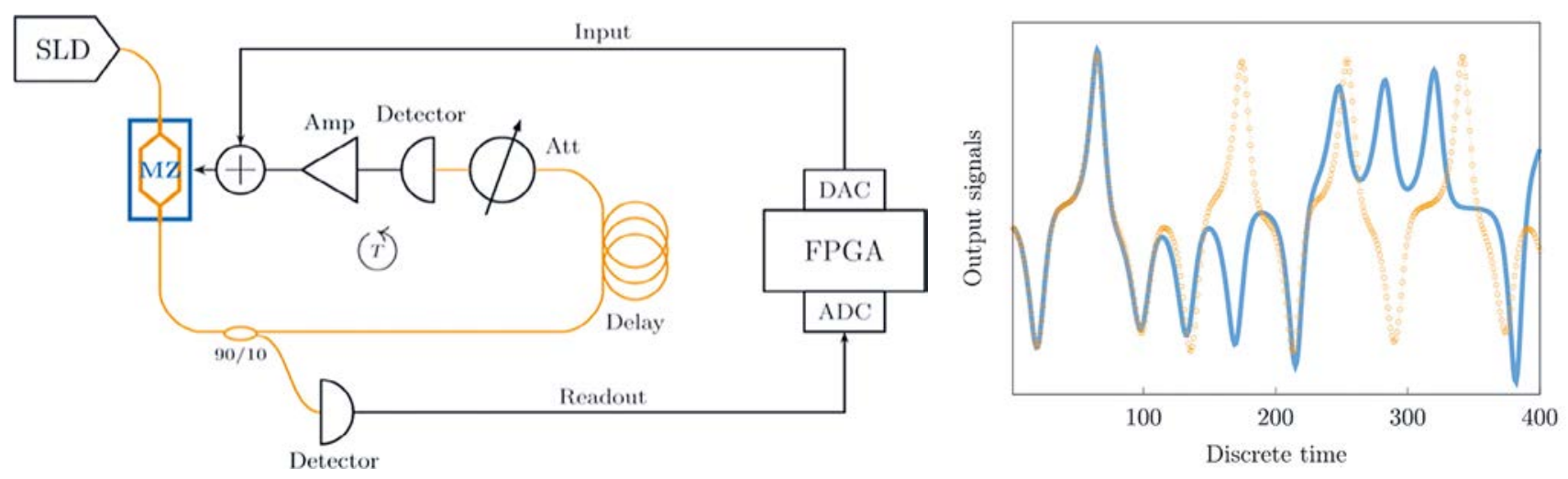

digits and to forecast chaotic time series. Subsequent work focused on increasing the speed of such systems and integrating them on a Photonic Integrated Circuit (PIC). Recently a compact and robust chip based reservoir was demonstrated by integrating a semiconductor laser and a $5.4 \mathrm{~cm}$ delay line (corresponding to a time delay of $\mathrm{T}=1.2 \mathrm{~ns}$ ) on an InP photonic chip [5]. This reservoir accommodates $\mathrm{N}=23$ neurons and showed good performance on tasks.

\section{USING RESERVOIRS TO}

\section{EMULATE DYNAMICAL SYSTEMS}

Let us imagine you would like to predict future samples of a time series, such as the evolution of the stock market, or the output of a dynamical system. Reservoir computing provides a very powerful way to achieve this goal [1]. All you need is a record of the time series during a sufficiently long period of time. First, you drive the reservoir with the recorded time series and train the reservoir to predict the time series one-time step ahead. When you reach the end of the recorded time series, you feed the one-time step ahead prediction back into the reservoir as input signal. In this way the reservoir becomes an autonomous system that can predict the time series a second time step ahead. Continuing like this, you will predict the long-time behaviour of the time series. Due to noise and limited precision your prediction will diverge after some time from the true time series, but it will be similar to the original time series. The reservoir, with its output feedback as input, is now emulating

Figure 3.

Reservoir computer used to emulate a chaotic system (adapted with permission from [6]). The optoelectronic reservoir is schematized on the left. Fiber optics components are in orange, electronic components are in black. The nonlinear node is a Mach-Zehnder modulator. The output is computed online using a Field-Programmable Gate Arrays (FPGA) coupled to an Analog-to-Digital Converter (ADC) and a Digital-to-Analog Converter (DAC), and then reinjected as input into the reservoir. Right, output of the reservoir when used to predict the trajectory of the Lorenz system. Initially (up to approximately $t=100$ ) the reservoir output (in orange) follows faithfully the trajectory of the Lorenz system. Afterwards the trajectories diverge, but stay similar to the trajectories of the Lorenz system.

the dynamical system you were trying to predict. This remarkable behaviour has been studied theoretically [1] and experimentally [6], see Fig. 3.

\section{CONCLUSION}

Reservoir computing, because of its versatility and ease of implementation, has allowed experimental implementations of neural networks with performances comparable to digital implementations. The architecture based on delay dynamical systems is behind much of this progress, as it simplifies the experimental system which consist only of a delay line and a non-linear node. However, such implementations have an inherent limitation, because all neurons are processed sequentially, which slows down overall operations. An important current objective is to develop architectures in which all neurons are processed simultaneously. Increasing speed, decreasing footprint, broadening the scope to other types of neural networks, are other important goals. Whether such analog neural electronic or photonic networks will find applications remains to be experienced. Today they are very exciting research topics, at the boundary between technology and artificial intelligence.
REFERENCES

[1] H. Jaeger, H. Haas, Science 304, 78 (2004)

[2] L. Appeltant, M.C. Soriano, G. Van der Sande et al., Nat. Commun. 2, 468 (2011)

[3] F. Duport, B. Schneider, A. Smerieri, et al., Opt. Express 20, 22783 (2012)

[4] D. Brunner, M.C. Soriano, C.R. Mirasso, et al., Nat. Commun. 4, 1364 (2013)

[5] K. Harkhoe, G. Verschaffelt, A. Katumba, et al., Opt. Express 28, 3086 (2020)

[6] P. Antonik, M. Haelterman, S. Massar, Phys. Rev. App. 7, 054014 (2017) 